\title{
Acknowledgements to reviewers
}

(c) The Japanese Society for Artificial Organs 2018

The Editors and Editorial Board Members are deeply grateful to the consultants listed below for their reviews of manuscripts from October 23, 2017, to October 30, 2018. The reviewers' generous guidance and recommendations are invaluable for us to maintain our journal's high standards of professionalism and quality and, most importantly, for the satisfaction and respect of our reading audience.

Arakawa, Mamoru
Abe, Yusuke
Akiyama, Masatoshi
Anai, Hirofumi
Ando, Masahiko
Ando, Ryoichi
Aokage, Toshiyuki
Baba, Shunsuke
Eguchi, Yutaka
Feng, Zhonggang
Firstenberg, Michael
Fuchimoto, Yasushi
Fujita, Tomoyuki
Fujiwara, Tatsuki
Fukuda, Makoto
Fukui, Toshihiro
Fukunaga, Kazuyoshi
Furukawa, Hiroshi
Furuzono, Tsutomu
Galli, Francesco
Haruguchi, Hiroaki
Hasegawa, Masahiro
Hata, Hiroki
Hibiya, Makoto
Higuchi, Chieko
Hiramatsu, Yuji
Hirata, Isao
Hirata, Mitsuhi
Hoshiba, Takashi
Hoshino, Masami
Ichiba, Shingo
Ichikawa, Hajime
Iguchi, Atsushi
Ijima, Hiroyuki

Isoyama, Takashi
Ito, Hiroshi
Iwasaki, Kiyotaka
Izumi, Yuichi
Janz, Viktor
Kakinoki, Sachiro
Kambe, Yusuke
Kanda, Keiichi
Kashiwa, Koichi
Kawaharada, Nobuyoshi
Kawahito, Koji
Kawanishi, Hideki
Kawasaki, Taku
Kimura, Mitsutoshi
Kimura, Tsuyoshi
Kinoshita, Osamu
Kinugawa, Koichiro
Kishida, Akio
Kitamura, Makoto
Kokubo, Kenichi
Komura, Makoto
Lee, WooSurng
Mahara, Atsushi
Maruyama, Osamu
Masakane, Ikuto
Masuzawa, Toru
Matsuda, Kenichi
Matsumine, Akihiko
Matsumoto, Kazuo
Mineshima, Michio
Mitamura, Yoshinori
Miyagawa, Shigeru
Miyasaka, Takehiro
Mizuno, Toshihide
Kandich

Sato, Koichi

Sezai, Akira

Shiba, Mariko

Shiga, Hidetoshi

Shiiya, Norihiko

Shime, Nobuaki

Shimizu, Keiki

Shiose, Akira

Sonoda, Hiromichi

Stronks, H Christiaan

Sudo, Akihiro

Sugano, Nobuhiko

Sumikura, Hirohito

Takaseya, Tohru

Takemasa, Ryuichi

Takewa, Yoshiaki

Tanaka, Akira

Tanoue, Yoshihisa

Tatsumi, Eisuke

Tayama, Eiki

Toda, Koichi

Togo, Konomi

Tokuhashi, Yasuaki

Tokunaga, Shigehiko

Tomo, Tadashi

Tsukiya, Tomonori

Usui, Akihiko

Yamamoto, Masaya

Yambe, Tomoyuki

Yano, Tetsuya

Yoshikawa, Yasushi

Yoshimi, Yasuo 